\title{
Combined Coronary Artery Bypass Grafting and Transcatheter Aortic Valve Replacement in a Patient with Severe Post-Radiation Damage
}

\author{
Yagel O\#, Eliaz R\#, Planer D, Lipey A, Rudis E, \\ Nassar H, Leibowitz D, Elitzur $Y$, Alcalai $R^{\#}$ and \\ Elbaz-Greener G* \\ Department of Cardiology, Hadassah Medical Center, \\ Hebrew University of J erusalem, Israel \\ \#Equally contributed to this work \\ *Correspondling author: Gabby Elbaz-Greener, \\ Department of Cardiology, Hadassah Medical Center, \\ Cardiology division, J erusalem, Room number 114, Israel
}

Received: May 31, 2021; Accepted: J une 18, 2021; Published: June 25, 2021

\begin{abstract}
Chest radiotherapy is a common treatment for mediastinal malignancy. The long-term effect of radiation can harm several of cardiovascular structures including pericardium, myocardium, valvular system, conduction system, and coronary arteries. Cardiovascular disease is the most common non-malignancy cause of death in radiation-treated patient, most often occurs many years after treatment. Valvular heart disease secondary to mediastinal radiation mainly affect the aortic and mitral valves. We present a unique case of 58 years old women with history of past mediastinal radiotherapy who presented with few episodes of true syncope, complete AV block, severe aortic stenosis and significant ostial left main and ostial right coronary artery disease.
\end{abstract}

Keywords: Radiotherapy; Aortic stenosis; Coronary ostial disease; Conduction abnormalities; TAVR; CABG; Hybrid procedure

\section{Ablbreviations}

BOOP: Bronchiolitis Obliterans with Organizing Pneumonia; TAVR: Transcatheter Aortic Valve Replacement; CABG: Coronary Artery Bypass Surgery; ECG: Electrocardiogram; LV: Left Ventricle; EF: Ejection Fraction; OP-CABG: Off-Pump Coronary Artery Bypass Graft; SAVR: Surgical Aortic Valve Replacement; AS: Aortic Stenosis; NYHA: New York Heart Association; PCI: Percutaneous Coronary Intervention

\section{Background}

Radiation therapy is used to treat several intrathoracic and chest wall malignancies. Radiation is an established mode of therapy for Hodgkin's disease in the last decades [1-3]. Most patients remain asymptomatic with generally subclinical radiation-related changes. The main determinants of post-irradiation changes are the radiation dose and exposure time [4]. Other determinants such as tissue vulnerability (fast proliferating tissues), area of exposure and repeated exposures are important as well [5]. The most common severe radiation-related pathologies are pneumonitis, lung necrosis, Bronchiolitis Obliterans with Organizing Pneumonia (BOOP), esophageal strictures, aortic and pulmonary trunk stenosis, occlusion or pseudoaneurysm. The typical cardiac radiation related damages are pericarditis, conduction system abnormalities (mainly atrioventricular blocks), valvular abnormalities and coronary artery disease in the form of calcifications and fibrotic occlusion of proximal segments, mainly involving the coronary ostia [1].

\section{Case Presentation}

A 58 years-old woman presented to the emergency department with two consecutive events of true syncope while resting. She mentioned that in the last few months she had chest pain on exertion. On arrival, her vital signs were normal. Neurological exam was unremarkable, physical examination revealed a harsh systolic murmur with diminished S2 and a weaken pulse.

The patient has history of Hodgkin's lymphoma at the age of 10 years old, that was treated with combined chemotherapy and highdose thoracic radiotherapy. Later, she was diagnosed with breast cancer at the age of 46 and underwent left mastectomy and adjuvant chemotherapy with Taxol and Herceptin. She also has a history of dyslipidemia and Hypertension, which is treated with a statin and ACE inhibitor.

On arrival lab test showed normal blood count and electrolytes with slight elevation of high sensitive troponin I. Electrocardiography (ECG) showed: sinus tachycardia with RBBB, normal PR and QT intervals, minimal ST segment elevation in AVR and ST segment depression in the lateral leads (Figure 1).

Chest X-ray showed porcelain aorta and pulmonary congestion (Figure 2). The patient was admitted to the Cardiac Intensive Care Unit (CICU) for further monitoring under presumed diagnosis of malignant syncope. During her monitoring in the CICU, she experienced another syncopal episode. ECG and monitor showed complete AV block with ventricular asystole of 7 seconds (Figure 3).

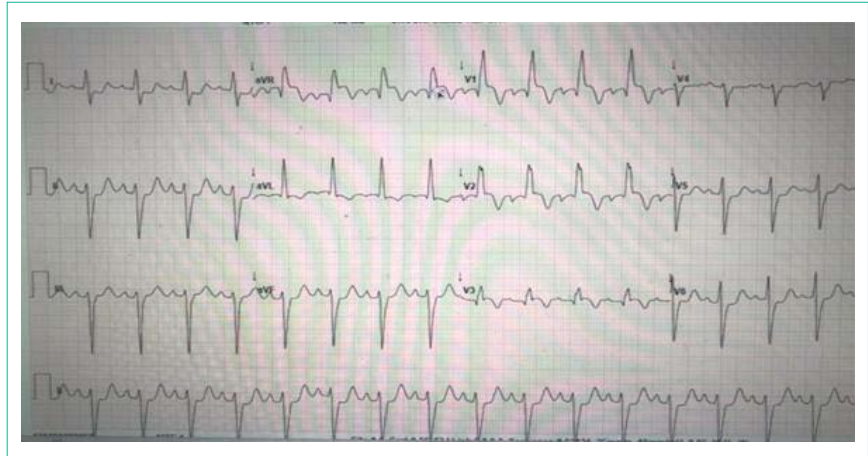

Figure 1: ECG.
Austin J Surg - Volume 8 Issue 3 - 2021

ISSN : 2381-9030 | www.austinpublishing group.com

Elbaz-Greener et al. @ All rights are reserved
Citation: Yagel O, Eliaz R, Planer D, Lipey A, Rudis E, Nassar H, et al. Combined Coronary Artery Bypass Grafting and Transcatheter Aortic Valve Replacement in a Patient with Severe Post-Radiation Damage. Austin J Surg. 2021; 8(3): 1273. 


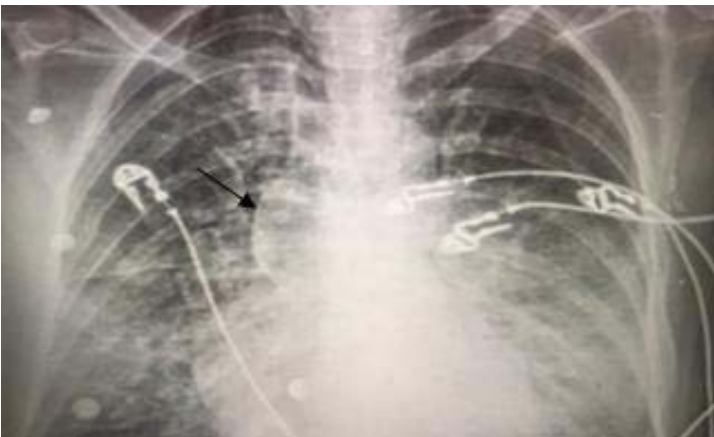

Figure 2: Chest X-ray.

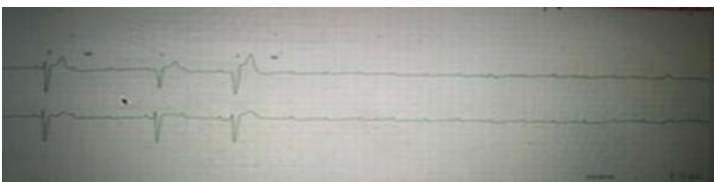

Figure 3: Cardiac monitor with complete AV block.

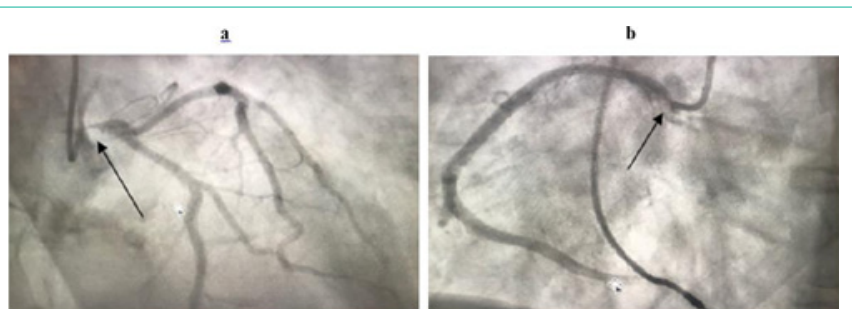

Figure 4: (a)-coronary angiogram with ostial left main disease. (b)-coronary angiogram with ostial right coronary artery disease.

An emergency temporary pacemaker was inserted.

An Echocardiogram showed-mild reduce Left Ventricle (LV) systolic function, Ejection Function (EF) of 50\%, severe Aortic Stenosis (AS) with a peak and mean gradients of $65 / 44 \mathrm{mmhg}$ respectively, Aortic Valve Area (AVA) of $0.5 \mathrm{~cm}^{2}$, moderate Aortic Regurgitation (AR), severe mitral annular calcification with moderate Mitral Stenosis (MS), moderate Tricuspid Regurgitation (TR) and moderate pulmonary hypertension (46mmhg TIG).

The patient was taken to the catheterization lab and underwent coronary angiography that demonstrated critical ostial left main (LM) coronary artery disease (Figure 4a). Left Anterior Descending (LAD) and Left Circumflex (LCX) arteries were patent without Atherosclerotic disease. Angiography of the right coronary artery (RCA) revealed also a significant ostial lesion (Figure 4b). During the procedure, a marked calcification of the ascending aorta was noted on fluoroscopy. At the end of the procedure the patient was stable with no chest pain or hemodynamic compromise.

A chest CT scan showed severely calcified ascending aorta (porcelain ascending aorta) with a diameter of $30 \mathrm{~mm}$ and no other major aortic branch disease (Figure 5).

An urgent heart team meeting was done to discuss the treatment options. Since her coronary disease was an aortic disease (aortoostial lesions) secondary to radiation damage and not the typical

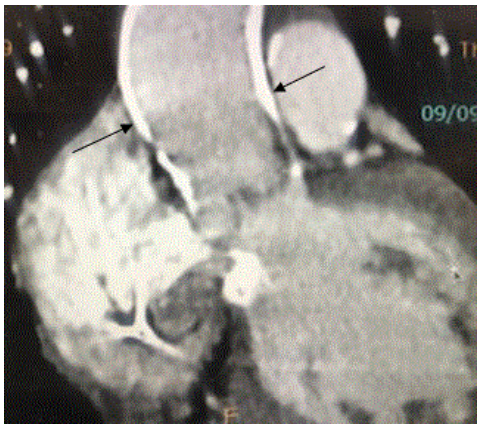

Figure 5: Chest CT scan with severely calcified ascending aorta (porcelain ascending aorta).

atherosclerotic disease, the impression was that there is a higher risk for long term stent restenosis and occlusion, hence CABG is preferable over PCI. As for the valvular disease, the heart team concluded that the patient was not a suitable candidate for Surgical Aortic Valve Replacement (SAVR) due to her porcelain aorta and a significant risk for stroke and aortic complications as dissection etc. The heart team decision was to perform a hybrid procedure of OffPump Coronary Artery Bypass Graft (OP-CABG) combined with Transcatheter Aortic Valve Replacement (TAVR).

CABG procedure was done off-pump with full revascularization with left internal mammary (LIMA) to LAD, Right Internal Mammary (RIMA) to RCA, RADIAL to Obtuse Marginal (OM) via T-graft from LIMA by the cardiac surgery team. After the CABG procedure, a TAVR was completed, with implantation of Evolute ${ }^{\mathrm{TM}}$ PRO 26 (Medtronic) in the aortic position.

The patient was extubated in the day after and was neurologically intact and hemodynamically stable. The patient underwent permanent pacemaker implantation and discharged from hospital in a general good condition. On 1-month follow up the patient was stable with NYHA 1-2.

\section{Discussion}

The patient presents with malignant syncope which could be secondary to each of her underlying cardiac conditions - the coronary disease, the valvular disease and the conduction disorder. This case encompasses and exemplifies the various pathologies which may be caused by thoracic radiation to the different mediastinal structures, notably the great vessels, heart valves and the conduction system.

To the best of our knowledge this is the first report of hybrid procedure of CABG and TAVR. The availability of transcatheter valvular therapies, advanced surgical techniques and hybrid Operating Rooms (OR) enables tailored hybrid therapies to treat complex patients utilizing the relative advantages in each method. The case demonstrates the change in the term "heart team" from discussion group to multidisciplinary task force working together in the OR.

\section{References}

1. Jaworski C, Mariani JA, Wheeler G, Kaye DM. Cardiac Complications of Thoracic Irradiation. Journal of the American College of Cardiology. 2013; $61 ; 2319-23282$. 
2. Wethal T, Lund MB, Edvardsen T, Fosså SD, Pripp AH, Holte H, et al. Valvula dysfunction and left ventricular changes in Hodgkin's lymphoma survivors. A longitudinal study. Br J Cancer. 2009; 101: 575-581.

3. Hancock SL, Tucker MA, Hoppe RT. Factors Affecting Late Mortality From Heart Disease After Treatment of Hodgkin's Disease. JAMA. 1993; 270: 1949-1955.
4. Rutqvist LE, Lax I, Fornander $\mathrm{T}$, Johansson $\mathrm{H}$. Cardiovascular mortality in a randomized trial of adjuvant radiation therapy versus surgery alone in primary breast cancer. Int J Radiat Oncol Biol Phys. 1992; 22: 887-896.

5. Gaya AM, Ashford RF. Cardiac complications of radiation therapy. Clin Oncol (R Coll Radiol). 2005; 17: 153-159. 